\title{
Random Telegraph Noise in Resistive Random Access Memories: Compact Modeling and Advanced Circuit Design
}

\author{
Francesco Maria Puglisi, Member, IEEE, Nicolò Zagni, Student Member, IEEE, Luca Larcher, \\ Member, IEEE, Paolo Pavan, Senior Member, IEEE
}

\begin{abstract}
In this work we report about the derivation of a physics-based compact model of Random Telegraph Noise (RTN) in $\mathrm{HfO}_{2}$-based Resistive Random Access Memory (RRAM) devices. Starting from the physics of charge transport, which is different in the high and low resistive states (HRS and LRS), we explore the mechanisms responsible for RTN exploiting a hybrid approach, based on self-consistent physics simulations and geometrical simplifications. Then, we develop a simple yet effective physics-based compact model of RTN valid in both states, which can be steadily integrated in state-of-the-art RRAM compact models. The RTN compact model predictions are validated by comparison with both a large experimental dataset obtained by measuring RRAM devices in different conditions, and data reported in the literature. In addition, we show how the model enables advanced circuit simulations by exploring three different circuits for memory, security, and logic applications.
\end{abstract}

Index Terms-RRAM; RTN; HRS; LRS; Compact Model; Variability; PUF; RNG; Verilog-A; Circuit Design

\section{INTRODUCTION}

$\mathrm{T}_{\mathrm{s}}$ HE relentless scaling of Complementary Metal Oxide Semiconductor (CMOS) technology is increasing the importance of phenomena associated with charge trapping/detrapping at discrete defect sites (either pre-existing in the virgin device or induced by stress), such as Stress-Induced Leakage Current (SILC) [1-3], Bias Temperature Instability (BTI) [4-6], and Random Telegraph Noise (RTN) [7-10]. Particularly, RTN represents a major reliability issue for conventional devices such as MOS transistors and their evolutions (e.g. FinFETs, GAA) [11-12], and for emerging devices such as the Resistive Random Access Memory (RRAM) [9-10]. In fact, RTN variability in RRAM can be wide enough to significantly shrink the read margin [9-10], especially in devices operated at low current levels for lowpower circuits. As RRAM technology is approaching the industrial stage, RTN mechanisms must be understood [7-10] and, possibly, mastered. This is essential to evaluate the technology potential and limitations for specific applications

Francesco Maria Puglisi, Nicolò Zagni, and Paolo Pavan are with the Dipartimento di Ingegneria "Enzo Ferrari", Università di Modena e Reggio Emilia, Via Vivarelli 10/1, 41125 Modena - Italy (phone: +39-059-2056324; fax: +39-059-2056329; e-mail: francescomaria.puglisi@unimore.it).

Luca Larcher is with the Dipartimento di Scienze e Metodi dell'Ingegneria, Università di Modena e Reggio Emilia, Via Amendola 2, 42122 Reggio Emilia - Italy. such as non-volatile memory (NVM) [13-16], neuromorphic computing [17-20], Physical Unclonable Function (PUF), and Random Number Generator (RNG) [21-23]. For instance, RTN effects are detrimental in multi-level NVM applications, reducing the read margin between adjacent bit distributions. $\mathrm{RTN}$ is an issue in neuromorphic circuits, in which it causes temporary and unpredictable random variations of the synaptic weight associated with the device. In addition, RNGs and PUFs performances can be adversely affected by RTN, worsening their figures of merit (e.g., randomness). Nevertheless, it has been suggested [24-25] to exploit the intrinsic randomness of RTN as an entropy source to realize RTN-based PUFs and RNGs, which could open new interesting perspectives. Finally, RRAM-based temperature sensors that exploit RTN have been proposed [26]. From the circuit designer perspective, it is therefore imperative to include RTN effects into RRAM models. Few attempts can be counted in the literature [27-30], and they typically address the phenomenon only in one of the two resistive states, i.e., either High- (HRS), or Low-Resistive State (LRS). Recently, we proposed a compact model for RTN in RRAM, valid in both resistive states, that correctly captures the statistics of the RTN properties (i.e., variations in amplitude and transition times) and the variability in the number of defects contributing to the RTN [31]. This model, implemented in Verilog-A, can be easily plugged in existing RRAM device models [13, 32-36], extending their potential. In this paper, we extend the work in [31] by including the details of the approach used to derive the compact model of RTN in HRS and LRS. In addition, we show how the proposed model enables: $i$ ) estimating the effect of RTN on the resistive states distribution; ii) evaluating how the randomness of a RRAM-based PUF circuit is affected by the presence of RTN; iii) designing the building block of an advanced RTN-based RNG circuit.

\section{DEVICES, EXPERIMENTS, AND SIMULATIONS}

\section{A. Devices and Experiments}

$\mathrm{TiN} / \mathrm{Ti} / \mathrm{HfO}_{2} / \mathrm{TiN}$ RRAMs with a $5 \mathrm{~nm}$ thick $\mathrm{HfO}_{2}$ layer are measured under different operating conditions [read voltage $\left(V_{R E A D}\right)$, reset voltage $\left(V_{R E S E T}\right)$, temperature $(T)$, current compliance $\left(I_{C}\right)$ ] (Fig. 1). After forming, each device is switched from the LRS to the HRS and back to the LRS 100 times and RTN is measured in both HRS and LRS at each cycle applying $V_{R E A D}$. This results in a wide dataset where the 

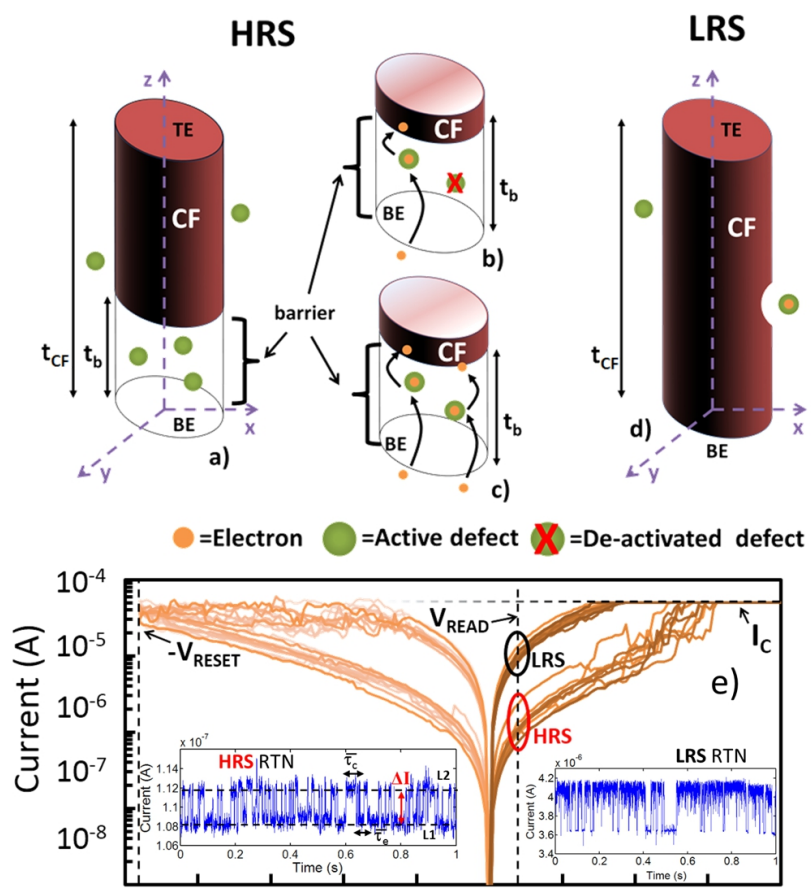

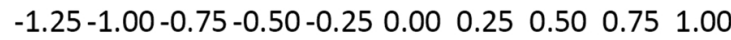
Voltage (V)

Fig. 1 - (a) Schematic picture of the device and (b-c) of the RTN mechanism in HRS, showing the defects (b) de-activation and (c) activation. Coordinates $(x-y-z)$, barrier thickness $\left(t_{b}\right)$, and CF thickness $\left(t_{o x}\right)$, are reported. (d) Schematic picture of the RTN mechanism in LRS, showing the electrostatic coupling between a charged defect and the conductive filament. (e) Experimental I-V curves measured at 20 consecutive switching cycles on a TiN/Ti/ $/ \mathrm{HfO}_{2} / \mathrm{TiN}$ device using $I_{C}=50 \mu \mathrm{A}$ and $V_{R E S E T}=1.2 \mathrm{~V}$, displaying cycle-to-cycle variability. $V_{R E S E T}$, (i.e. the maximum absolute value of the voltage during the reset sweep), $I_{C}$, and $V_{R E A D}$ are highlighted. Insets in (e) show a two-level RTN, related to a single trap, as detected in HRS (left) and in LRS (right). In the left plot, the statistical RTN parameters are evidenced [(i.e., the average capture time $\left(\overline{\tau_{c}}\right)$, the average emission time $\left(\overline{\tau_{e}}\right)$, the fluctuation amplitude, $\left.(\Delta I)\right]$.

effects of RTN fluctuations are superimposed to the device-todevice and cycling variability. All the experiments are performed with a Keithley 4200-SCS, with the temperature being controlled by a dedicated thermo-chuck. The analysis of the RTN signals is performed by means of the Factorial Hidden Markov Model (FHMM) [37-38]. This statistical method allows decomposing the multi-level RTN signal into a superposition of two-level signals (i.e., components), each attributed to the activity of an individual defect. This allows correctly estimating the properties of each component from the complex multi-level RTN signal. These properties [i.e., the fluctuation amplitude $(\Delta I)$, the average capture $\left(\bar{\tau}_{c}\right)$ and average emission $\left(\bar{\tau}_{e}\right)$ times (inset of Fig. 1(e))] can be linked to the physical properties of the associated defect.

\section{B. Simulations}

Charge transport and RTN fluctuations are simulated using MDLab's Ginestra software [39], which accounts for the 3-D potential and temperature maps in the whole RRAM device, as well as the direct and the multi-phonon trap-assisted tunneling (DT and MP-TAT, respectively) charge transport [40]. The lattice relaxation and the electron-phonon coupling associated with the charge trapping at defect sites are also included. This allows accounting for the atomic rearrangement around the defect occurring upon the charge trapping that strongly affects the charge trapping and de-trapping rates and the localized power dissipation at the defect site [40].

\section{Charge Transport IN $\mathrm{HFO}_{2}$ RRAM}

Developing a compact model for RRAM devices that includes the effects of RTN requires understanding the physical mechanisms dominating charge transport in the two resistive states. Indeed, in general, the RTN is a temporary alteration of the current flow [41], determined by different physical mechanisms in HRS and LRS [30, 41-42]. Therefore, it is mandatory to discuss the charge transport mechanism and its alterations separately for the two states.

\section{A. Charge Transport in LRS}

In LRS, the device is characterized by the presence of a full conductive filament (CF) shunting the two electrodes (Fig. 1d). It is accepted that the $\mathrm{CF}$, created during the preliminary forming operation, is formed by tightly packed oxygen vacancy defects, and its size is controlled by the current compliance, $\mathrm{I}_{\mathrm{C}}$, used in the forming and set operations [30-36, 42]. In this resistive state, charge transport is due to drift of de-localized electrons drift in the CF [30-36, 42], consistently with the ohmic-like behavior generally observed in LRS. This charge transport mode is due to the nature of the $\mathrm{CF}$, associated with the formation of a conductive sub-band [42].

\section{B. Charge Transport in HRS}

The reset operation, driving the device in HRS, leads to the partial re-oxidation of the $\mathrm{CF}$, creating a dielectric barrier [30$36,42]$, as shown in Fig. 1(a-b-c). The dielectric barrier thickness $\left(t_{b}\right)$ is determined by the reset conditions (Fig. 1), i.e., $V_{R E S E T}$, and $T$. The HRS current is dominated by the electron MP-TAT at positively charged oxygen vacancy defects $\left(\mathrm{VO}^{+}\right)$in the re-oxidized tip of the $\mathrm{CF}$ (dielectric barrier in Fig. 1(a-b-c)) [32-33, 40-42].

\section{THE RANDOM Telegraph NoISE Model}

A complete RTN compact model must capture the inherent variability in the number of defects contributing to the RTN and in their statistical properties $\left(\Delta I, \overline{\tau_{c}}, \overline{\tau_{e}}\right)$. These all depend on the operating conditions and on the physical state of the device (i.e. HRS or LRS). In the following, we show how these features can be implemented in the model.

\section{A. RTN Amplitude Statistical Model in HRS}

The physical mechanism responsible for RTN in HRS is still under debate [43-46], but it is widely believed that is due to the temporary (de-)activation of $\mathrm{VO}^{+}$defects, distributed in the barrier, assisting charge transport [29-30, 43, 46], as sketched in Fig. 1(b-c). The mechanisms leading to (de)activation is also due to charge (de-)trapping in additional slow defects that do not participate to charge transport (supposedly oxygen interstitial atoms) [29-30, 43]. To gain further insights, we perform physics-based simulations of charge transport across a $10 \times 10-\mathrm{nm}^{2}$ defect-rich $\mathrm{HfO}_{2}$ dielectric barrier with TiN electrodes (Fig. 2). Simulations are performed considering different applied voltage, barrier 

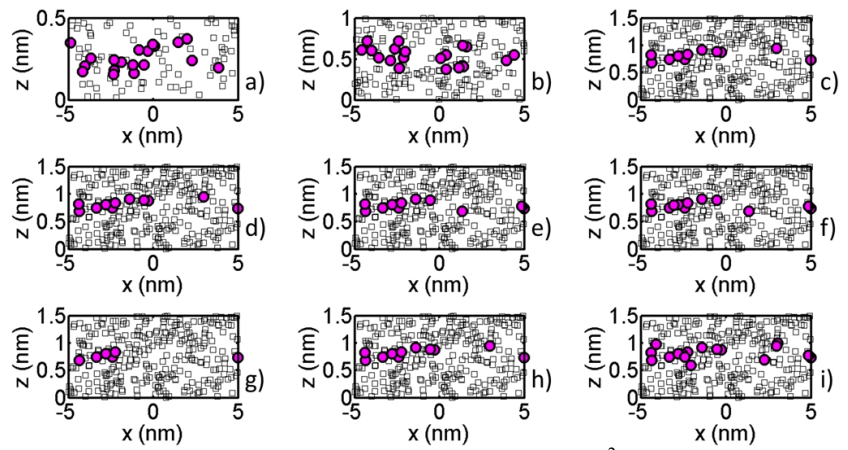

Fig. 2 - Simulation of charge transport in a $10 \times 10 \mathrm{~nm}^{2}$ barrier. Projection of the $3 \mathrm{D}$ defects distribution on the $\mathrm{x}-\mathrm{z}$ plane, see Fig. $1 . \mathrm{Vo}^{+}$defects (black squares) are randomly distributed in the barrier with a density of $N_{T}=2 \cdot 10^{21}$ $\mathrm{cm}^{-3}[29-30,43,47]$. Few $\mathrm{Vo}^{+}$defects (violet circles) in the middle of the barrier $( \pm 0.2 \mathrm{~nm})$ assist $>95 \%$ of the total current, regardless of the operating conditions. (a-b-c) $t_{b}=0.5 \mathrm{~nm}-1.0 \mathrm{~nm}-1.5 \mathrm{~nm}$ at $V_{R E A D}=100 \mathrm{mV}$ and $T=$ $25^{\circ} \mathrm{C}$. (d-e-f) $t_{b}=1.5 \mathrm{~nm}$ at $V_{R E A D}=100 \mathrm{mV}$ and $T=25^{\circ} \mathrm{C}-75^{\circ} \mathrm{C}-125^{\circ} \mathrm{C}$. (g-h-i) $t_{b}=1.5 \mathrm{~nm}$ at $V_{R E A D}=50 \mathrm{mV}-100 \mathrm{mV}-150 \mathrm{mV}$ and $T=25^{\circ} \mathrm{C}$.

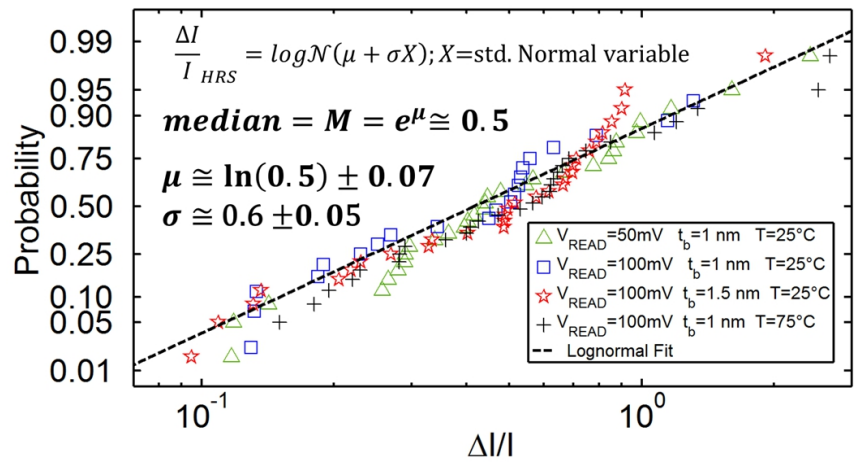

Fig. 3 - Distribution of simulated $\Delta I / I$ in HRS obtained using kinetic MonteCarlo simulations. The I-time traces related to a $10 \times 10 \mathrm{~nm}^{2}$ MIM device with TiN electrodes with a $1.0 \mathrm{~nm}$-thick barrier are simulated, including the effect of $\mathrm{VO}^{+}$defects activation and de-activation. $\mathrm{Vo}^{+}$are randomly distributed in the barrier with a density of $N_{T}=2 \cdot 10^{21} \mathrm{~cm}^{-3}$ [29-30, 43, 47]. Simulations are performed in different conditions $\left(V_{R E A D}, t_{b}\right.$, and $\left.T\right)$ and are repeated on 10 different device realizations by randomizing defects positions and energies (a total of 40 simulation runs). $\Delta I / I$ distribution is insensitive to operating conditions in agreement with experimental reports [29-30, 43]. Its statistical properties ( $\mu$ and $\sigma$, with related uncertainties) are reported in the figure.

thickness, and temperature values. The presence of defects and of the trapped charge is self-consistently accounted for as described in Section II-B. For each simulation, we consider randomly distributed defects (using uniform spatial and energetic distributions [40]), and we estimate the $\Delta I / I_{H R S}$ by de-activating an individual, randomly selected, defect. In this context, $\Delta I$ is defined as the current contribution given by an individual $\mathrm{VO}^{+}$defect when active [29-30] (Fig. 1(b-c)), while the overall current, $I$, is the sum of all the current contributions. It is evident from Fig. 2 that a small fraction (violet circles) of all the $\mathrm{Vo}^{+}$defects in the barrier (black squares) is responsible for almost the whole HRS current (i.e., $>95 \%$ ), regardless of $t_{b}$ (determined by reset conditions), $V_{R E A D}$, and $T$. This is because the current assisted by a defect is maximized when the defect has small and comparable $\overline{\tau_{c}}$ and $\overline{\tau_{e}}$, which is likely for the defects in the middle of the barrier. As such, since RTN is due to the random (de-)activation of these defects, the average $\Delta I / I_{H R S}$ (or equivalently $\Delta R / R_{H R S}$ ) is expected to be constant in every operating condition. Moreover, due to the random nature of the defects (de- )activation, it is unlikely for the defects to be simultaneously all active or inactive, suggesting an average $\Delta I / I_{H R S}<1$. Naturally, if an individual defect experiencing activation and de-activation drives more than half of the overall current (when active), then it will be possible for $\Delta I / I_{H R S}$ to be $>1$. Devising a simple average formulation for the RTN amplitude in HRS requires estimating the average $\Delta \mathrm{I}$ related to the (de)activation of an individual defect, which strongly depends on the defect location, energy, cross-section, as well as on the local voltage and temperature profiles. Results are reported in Fig. 3, where the probability distribution of the resulting $\triangle I / I_{H R S}$ appears lognormal and invariant with operating conditions, in agreement with previous experimental reports [29-30, 43]. This lets writing:

$$
M\left(\frac{\Delta R}{R}_{H R S}\right) \cong \frac{1}{2} \quad \sigma\left(\frac{\Delta R}{R}_{H R S}\right) \cong 0.6
$$

Hence, despite the significant intrinsic variability and the complexity of the RTN mechanism, it is possible to conceive a simple statistical description of RTN fluctuations amplitude in HRS in every operating condition.

\section{B. RTN Amplitude Statistics Model in LRS}

In LRS, RTN is commonly attributed to electron trapping and de-trapping at individual defect sites in the proximity of the CF [i.e. within one electron Debye length $(\lambda)$ ] [44, 48-50]. The trapped charge perturbs the potential in its surroundings causing a screening effect on the portion of the CF close to the defect, which induces a resistance change (Figs. 1(d) and 4). As opposite to the HRS, the relative resistance change $\left(\triangle R / R_{L R S}\right)$ depends on the $\mathrm{CF}$ geometry, i.e. its radius $\left(r_{C F}\right)$ (assuming a cylindrical CF), and its thickness $\left(t_{o x}\right)$. For a large $\mathrm{CF}$ (i.e. $r_{C F}>>\lambda$ ) the $\mathrm{CF}$ screened section is much smaller than the $\mathrm{CF}$ area, $S_{C F}=\pi \cdot r_{C F}{ }^{2}$, causing a relatively small resistance change $[44,48-50]$. As the CF gets smaller, the screened portion of the $\mathrm{CF}$ gets comparatively wider, with a larger impact on the average relative resistance (or, equivalently, current) change, as also reported in the literature [44, 48-50]. However, while the average effect is dependent on the CF size, the deviations from the average are CF-size independent, as they only depend on the distance between the defect and the $\mathrm{CF}$ edge. This results in the lognormal $\triangle I / I_{L R S}$ distribution having a slope, i.e., $\sigma$, of about 0.3 [44] that is invariant with the CF size (in turn controlled by $I_{C}$ ) [32-36]. Conversely, the median $\triangle I / I_{L R S}$ value changes with the $C F$ size:

$$
M\left({\frac{\Delta R}{R_{L R S}}}\right)=f\left(S_{C F}\right) \quad \sigma\left({\frac{\Delta R}{R_{H R S}}}\right) \cong 0.3
$$

Therefore, once the relation between $S_{C F}$ and the median $\Delta I / I_{L R S}$ is found, it is possible to estimate the $\Delta I$ of any RTN current fluctuation regardless of the resistive state and the operating conditions. For this reason, we will focus on the description of $f\left(S_{C F}\right)$. To this point, performing physics-based simulations in LRS would result in a huge computational burden due to the presence of the full CF. Instead we exploit the $\mathrm{CF}$ geometry to calculate the relative resistance change $\Delta R / R_{L R S}$ induced in the CF by a charge trapped at a close defect site. Still, $\Delta R / R_{L R S}$ depends on many variables: i) the distance between the defect and the CF edge; ii) the local $\mathrm{CF}$ cross-section and its composition; iii) the effective screening 

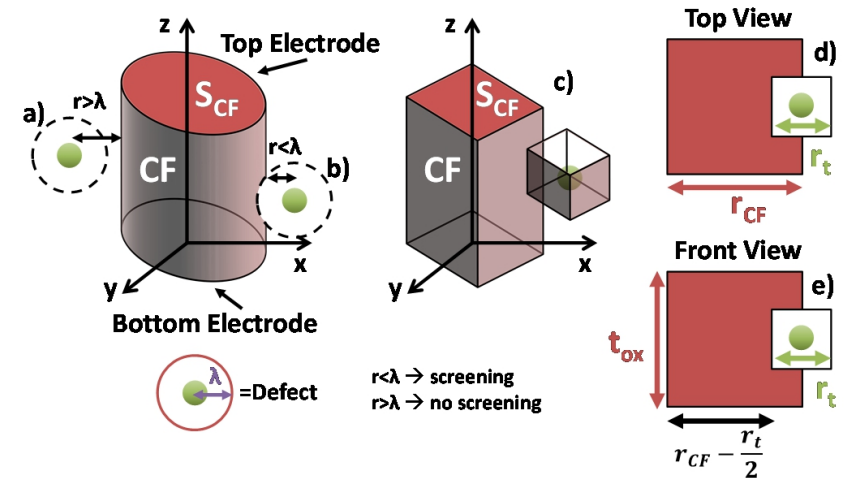

Fig. 4 - Schematic of RTN mechanism in LRS. Defects close to the CF $(r<\lambda)$ cause a screening effect on a portion of the $\mathrm{CF}$, inducing a resistance change. (a) No screening $(r>\lambda)$. (b) Screening on a wide-area CF. (c) Simplified geometrical framework with (d) its top view and (e) its front view.

length of an electron in a complex medium; iv) the coupling between the electric field of the trapped electron and a portion of the CF. Therefore, a compact formulation can be obtained only by introducing some reasonable simplifying assumptions. We assume an ohmic-like charge transport in the CF, justified by the linear I-V relation typically observed in LRS (Fig. 1(e)), and a uniform CF composition, i.e., a constant CF resistivity $\left(\rho_{C F}\right)$ in space. In addition, we consider the $\mathrm{CF}$ geometrically uniform (i.e., a prism-shaped CF with arbitrary base profile). Though the exact shape of the $\mathrm{CF}$ is currently under debate in the scientific community, this assumption is extremely useful in easing calculations and is frequently adopted [32-36]. To simplify the problem, here we assume an individual defect site placed at the edge of the CF (i.e. we assume zero distance between the defect and the CF edge), as shown in Fig. 4. This defect site, when filled with an electron, exhibits the maximum screening effect on the CF. Moreover, we consider that the trapped charge produces uniform screening in a spherical region around itself, and no screening beyond this region. In addition, we consider the screened portion of the $\mathrm{CF}$ to be completely unavailable to charge transport (i.e. we consider its resistance to be infinite). The full CF resistance with no screening effect, $\left(R_{L R S}\right)$, and the relative resistance change induced in the $\mathrm{CF}$ by the trapped charge $\left(\Delta R / R_{L R S}\right)$, can be written as:

$$
R_{L R S}=\frac{\rho_{C F} \cdot t_{o x}}{S_{C F}} \quad \frac{\Delta R}{R_{L R S}}=\frac{R_{L R S}{ }^{*}-R_{L R S}}{R_{L R S}}
$$

where $R_{L R S}{ }^{*}$ is the resistance of the CF when the screening effect occurs. In the case of a cylindrical filament, calculating this term is complex, as it requires two difficult steps. The first requires calculating the intersection volume between a cylinder (i.e., the CF) and a sphere (i.e., the electric field of the trapped charge). This can be accomplished by using an analytic expression that requires solving numerically elliptic integrals [51], which makes its implementation tedious. The second is the calculation of the resistance of an irregular 3D structure, which may require numerical techniques. Here, to simplify calculations (Fig. 4(c-d-e)), we consider the CF to be a prism with a square base and cross-section $S_{C F}$, i.e., side $r_{C F}$. This allows rewriting eq. (3) as $R_{L R S}=\rho_{C F} \cdot t_{o x} / r_{C F}{ }^{2}$. In addition, we simplify the spherical electric field of the trapped charge to a cube with side $r_{t}$, a parameter accounting for the effective screening length of the trapped charge in this simplified framework, related to $\lambda$ [44, 48-50]. It is worth noting that $r_{t}$ can be used as a fitting parameter, effectively compensating for the assumption of zero distance between the $\mathrm{CF}$ and the trapped charge. Under these conditions, the $\Delta R / R_{L R S}$ in eq. (3) can be written in terms of $r_{t}$ and of the CF properties $\left(t_{o x}, r_{C F}, \rho_{C F}\right)$ :

$$
\frac{\Delta R}{R_{L R S}}=\frac{\left[\frac{\rho_{C F} \cdot\left(t_{o x}-r_{t}\right)}{r_{C F}{ }^{2}}+\frac{\rho_{C F} \cdot r_{t}}{r_{C F}{ }^{2}-\frac{r_{t}{ }^{2}}{2}}\right]-\frac{\rho_{C F} \cdot t_{o x}}{r_{C F}{ }^{2}}}{\frac{\rho_{C F} \cdot t_{o x}}{r_{C F}{ }^{2}}}
$$

Some mathematical operations allow rewriting eq. (4) as:

$$
{\frac{\Delta R}{R_{L R S}}}=\frac{r_{t}{ }^{3}}{2 t_{o x} \cdot\left(r_{C F}{ }^{2}-\frac{r_{t}{ }^{2}}{2}\right)} \cong \frac{r_{t}{ }^{3}}{2 t_{o x} \cdot r_{C F}{ }^{2}}=\frac{r_{t}{ }^{3}}{2 t_{o x} \cdot S_{C F}}
$$

which is the formula to estimate the relative resistance change (or equivalently, relative current change, $\Delta I / I_{L R S}$ ) induced in the CF by a trapped charge at a close defect site. In the last passage, $r_{C F}^{2} \gg r_{t}^{2} / 2$ was assumed.

\section{RTN Capture and Emission Times}

The complete statistical model for the RTN fluctuation amplitude developed so far must be accompanied by a model to estimate $\tau_{c, e}$ of each defect contributing to the RTN. This is necessary to reproduce the RTN signals over time, allowing transient simulations to be performed with the proposed compact model. Since the physical mechanism causing RTN is associated with charge (de-)trapping in both resistive states, we calculate for each defect the related $\overline{\tau_{c}}$ and $\overline{\tau_{e}}$ using the MP-TAT compact formalism adopted in [30-31, 52]. These formulae, described in [52], require defining $V, T$, and the defect distance from the electrodes $(d)$. In turn, the latter is determined by the defect vertical position within the dielectric barrier when the device is in HRS, or alongside the CF within the whole insulating layer when the device is in LRS). Since charge carriers can be captured from (and emitted to) either the top or the bottom electrodes, $\bar{\tau}_{c}$ and $\bar{\tau}_{e}$ are calculated for both cases (two possible capture and two possible emission processes), and the minimum value is considered, as it is representative of the most likely event between the two. In

$$
\tau_{C} \propto e^{\left(\frac{d}{\lambda_{c}}\right)} e^{\left(\frac{E_{C}(V)}{k T}\right)} ; \tau_{e} \propto e^{\left(\frac{d}{\lambda_{e}}\right)} e^{\left(\frac{E_{e}}{k T}\right)}
$$

addition, it is necessary to specify a few defect properties (e.g., thermal ionization energy, relaxation energy) effectively lumped in the typical capture $\left(\lambda_{c}\right)$ and typical emission $\left(\lambda_{e}\right)$ lengths, and the capture $\left(E_{c}\right)$ and emission $\left(E_{e}\right)$ activation energies that only depend on the defect typology [30-31, 40, 52] (mainly oxygen ions in HRS and $\mathrm{Vo}^{+}$in LRS). These parameters are calculated exploiting ab-initio calculations (i.e. Density Functional Theory or Molecular Dynamics), see [30$31,40,52]$ and references within.

\section{Model Validation and Implementation}

The compact model proposed here has been validated by comparison with an extensive experimental dataset (Fig. 5). Five devices with a $5 \mathrm{~nm} \mathrm{HfO}$ layer were formed at different $I_{C}$ values (ranging from $40 \mu \mathrm{A}$ to $150 \mu \mathrm{A}$ ), and then cycled through HRS and LRS for 100 times to collect RTN data in 


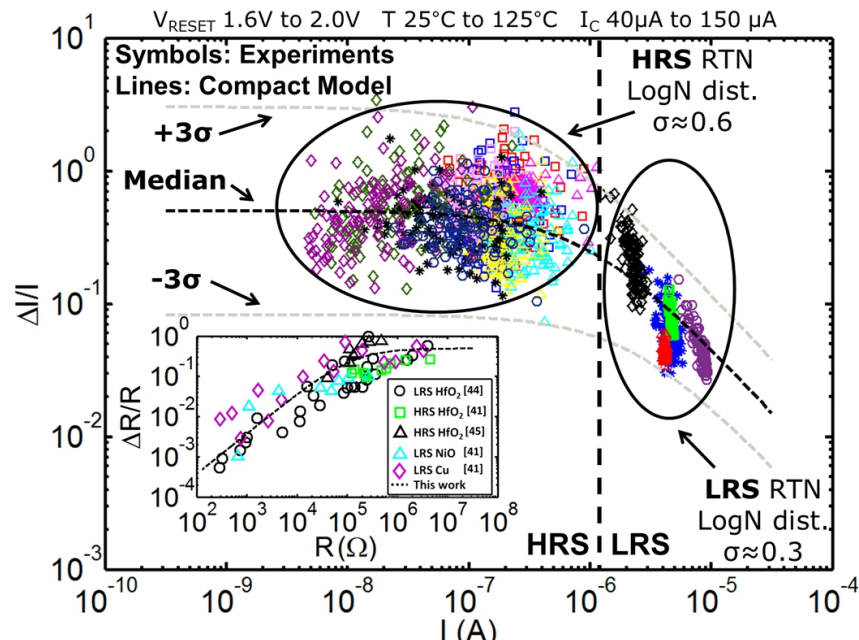

Fig. 5 - Experimental $\Delta I / I$ vs. $I$ (symbols) in HRS (left) and LRS (right) at different operating conditions ( $\left.I_{C}, V_{R E S E T}, T\right)$ for five devices (different symbols) with a $5 \mathrm{~nm} \mathrm{HfO}_{2}$ layer. Model predictions (dashed lines) are in excellent agreement with real data. The extracted $\sigma$ values of the normal distributions associated with the lognormal $\Delta I / I$ distributions in HRS and LRS agree with the values extracted from simulations and used in the compact model (the $\pm 3 \sigma$ predictions are also reported as grey dashed lines). Inset - The compact model predictions plotted as $\Delta R / R$ vs. $R$ (dashed line) agree with RTN data in HRS and LRS taken from the literature (symbols) [41, 44-45] Data from devices based on different materials (i.e. $\mathrm{NiO}$ and $\mathrm{Cu}$ ) are also reported, showing a good agreement with the model predictions.

both resistive states, also accounting for the effects of cycleto-cycle and device-to-device variability, see Section II-A. In addition, the experiment was repeated for all devices at different $V_{R E S E T}$ and $T$. The experimental $\Delta I / I$ vs. $I$ scatter plot is reported in Fig. 5 along with the compact model prediction, which is found to be in excellent agreement with experimental data. The extracted $\sigma$ values of the $\Delta I / I$ distributions in HRS and LRS agree with the values extracted from simulations and used in the compact model. This is further confirmed by the fact that almost the entire experimental dataset falls within the $\pm 3 \sigma$ predictions reported as grey dashed lines in Fig. 5 . Moreover, the compact model prediction is also in excellent agreement with data taken from recent literature for $\mathrm{HfO}_{2}$ RRAM and for devices made of different materials (e.g. $\mathrm{Cu}$ and NiO RRAM) [41, 44-45]. This is consistent with the compact model not depending on the CF resistivity, and suggests that the same (or a similar) RTN mechanism may hold for devices employing different materials. In this respect, it is noteworthy that $r_{t}$ can be used, with the other parameters unchanged, to compare the "strength" of the RTN in LRS in RRAM devices made of different materials. The $r_{t}$ value used in our simulations is $r_{t} \approx 1.7 \mathrm{~nm}$, comparable to $\lambda$ [48]. Moreover, reproducing the $\Delta I / I$ vs. $I$ curves allows estimating $\rho_{C F}$ from eq. (3-5), which assumes a value of $\approx 10 \mathrm{k} \Omega \cdot \mathrm{nm}$, in agreement with values reported in the literature [30, 42-43]. In addition, the inherent ability to deal with multiple defects allows the model to reproduce the $1 /$ f noise resulting from the superposition of many RTN signals [28, 30, 43].

The compact model proposed here can be effortlessly integrated into existing RRAM device compact models [32$36]$, as it requires very few inputs that are easily provided by compact device models, i.e. $t_{o x}$, the device resistance $(R), V, T$, the current simulation time, $t, S_{C F}$, and $t_{b}$ (Fig. 1). The algorithm has been implemented in Verilog-A, and tested
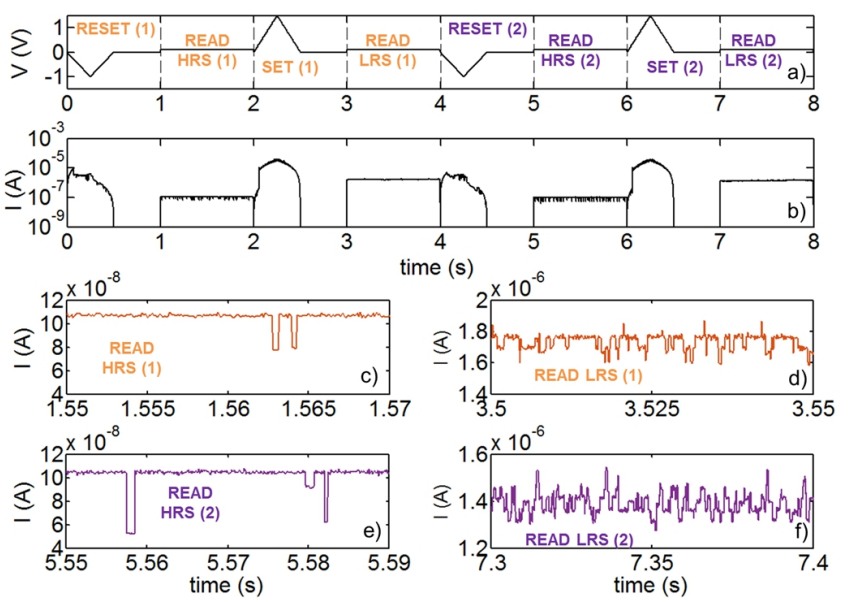

Fig. 6 - Simulations of resistance switching and RTN readout in HRS and LRS. An RRAM with $R_{L R S} \approx 15 \mathrm{k} \Omega$ is considered. The compact device model used in this simulation [32-33] has been extended with the RTN module. The voltage waveform in (a) is applied to perform the reset $\left(V_{R E S E T}=1 \mathrm{~V}\right)$, the set $\left(V_{S E T}=1.5 \mathrm{~V}\right)$, and the readout operation $\left(V_{R E A D}=100 \mathrm{mV}\right)$ for two consecutive cycles. The readout current (b) shows RTN signals in both states, zoomed in (c-f). The cycle-to-cycle variability of RTN is correctly accounted for, as highlighted by the different RTN patterns obtained in the same resistive states in two consecutive cycles.

using an existing RRAM device model [32-33], extended with the proposed RTN model. The results of RTN readout simulations in HRS and LRS are reported in Fig. 6. The details of its implementation are described in details elsewhere [31].

\section{ApPliCATIONS AND Circuit DESIGN}

The proposed model allows considering the presence of RTN in circuit simulations, which is advantageous in the design of circuits for many applications. Here we discuss some examples: a) RTN-aware design of RRAM memory circuits and b) PUFs, c) design of RTN-based RNGs.

\section{A. RTN-aware Design of Multi-bit Memory Circuits}

In the design of a RRAM array for memory application, it is imperative to guarantee an adequate margin between adjacent bit distributions. Nevertheless, the presence of RTN reduces the read margin between neighboring distributions, especially if fast read operation is required (i.e., zero or small averaging time). Therefore, a compact model that can account for the effects of RTN is highly desirable, especially for the design of multi-bit cell arrays. This is clarified in Fig. 7, where we report the comparison between simulations that include and exclude the effects of RTN, respectively. We simulated 100 full switching cycles of an individual cell, repeating the simulation for two different $V_{\text {RESET }}$ values, namely $1.0 \mathrm{~V}$ and 1.6 V. This allows building the HRS resistance distributions, accounting for the cycling variability. The read current is detected at each cycle by applying $V_{R E A D}=100 \mathrm{mV}$ using an averaging time of $100 \mu \mathrm{s}$. Regardless of the reset condition, the distribution with RTN is clearly more stretched than the one without RTN. Tail states appear due to RTN fluctuations, which can be detrimental for multi-bit memory applications as they reduce the margin between adjacent bit distributions, potentially leading to read failures. In the example of Fig. 7, simulations not accounting for the RTN would predict a tiny but non-zero margin between the two obtained distributions using $V_{\text {RESET }}=1.0 \mathrm{~V}$ and $V_{\text {RESET }}=1.6 \mathrm{~V}$. Nevertheless, our 


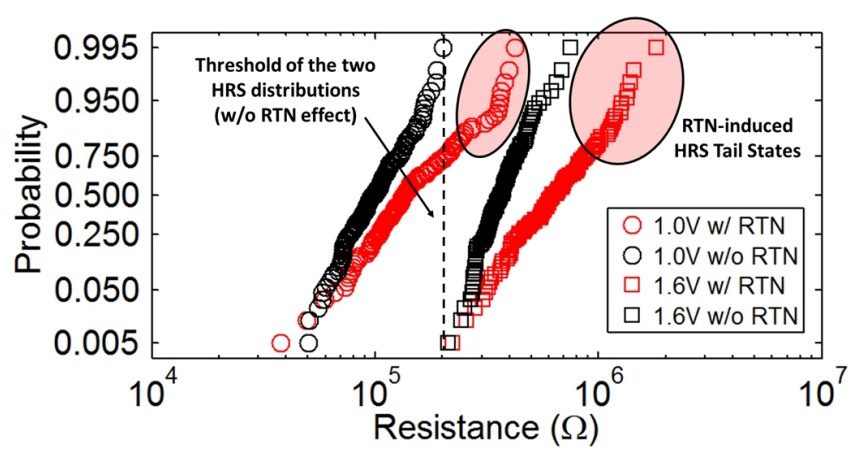

Fig. 7 - HRS resistance (read at $V_{R E A D}=100 \mathrm{mV}$ ) distributions obtained by cycling an individual cell 100 times with (red symbols) and without (black symbols) RTN, for $V_{\text {RESET }}=1.0 \mathrm{~V}$ (circles) and $1.6 \mathrm{~V}$ (squares). The presence of RTN broadens the resistance distribution and results in tail states as well as deviations from a pure lognormal trend.

simulations show that these two reset conditions cannot be safely assigned to two distinct logic states due to their RTNinduced overlap, which would lead to failures. This effectively reduces the number of bits that can be stored in a cell.

\section{B. The impact of RTN on a 128-bit RRAM-based PUF}

The stochastic behavior and intrinsic variability of RRAM devices can be exploited to realize PUF circuits for hardware security [21-23] (Fig. 8(a)). The performance of RRAM-based PUF circuits strongly depends on RRAM reliability, severely affected by noise issues and read instabilities [23]. This is particularly true when HRS is chosen to implement PUF because of the wider resistance distribution and of the lower power consumption than in LRS [23]. Consequently, it is important to deploy compact models able to evaluate the performance of PUF circuits in the presence of RTN. The effect of RTN on the performance of a 128-bit RRAM-based PUF is here investigated briefly. Initially, an individual cell is instantiated in LRS, with $I_{C}=100 \mu \mathrm{A}$. Then the cell is cycled 100 times between HRS and LRS, using a $1 \mathrm{~ms}$ ramped voltage down to $-1.7 \mathrm{~V}$ for the reset operation. When the cell is in the HRS, the current at $V_{R E A D}=100 \mathrm{mV}$ is detected, allowing the estimation of the median value of the current in HRS that will serve as reference. A 128-bit PUF circuit is then instantiated and all the devices are initially set to the LRS, and then driven to the HRS. The information related to the resistive state of the 128-bit string of devices is then digitized by comparing each device's current with the reference one. Obviously, when the RTN is not considered, the read current is constant over time but it may strongly vary over time when RTN is considered. Therefore, the detected value of the read current depends on the adopted reading scheme (Fig. 8(b)). In this work, we evaluated the performance of the PUF circuit and the effect of RTN performing the read operation with two different averaging time values, $1 \mathrm{~s}$ and $100 \mu \mathrm{s}$, respectively. The randomness of the PUF circuit (i.e., the percentage of logic " 0 "s or " 1 "s in the response) is assessed for 100 responses. Simulations without considering RTN (Fig. 8(c,d)), result in a randomness distribution centered almost at $50 \%$, as expected [21-23]. Nevertheless, when the effect of RTN is considered, the randomness distribution may be significantly shifted, depending on the reading scheme. A long averaging time (e.g., $1 \mathrm{~s}$ ) reduces the probability of bit flipping, smearing out the effect of wide RTN fluctuations (Fig. 8(c)).

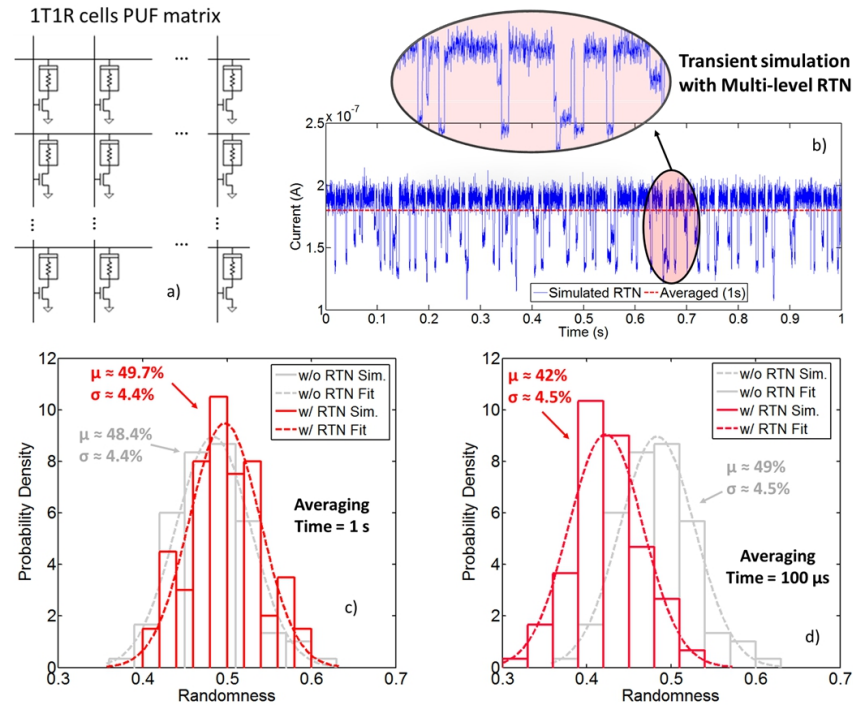

Fig. 8 - (a) Schematic of the 1T1R cells implemented in PUF matrix. (b) Simulated RTN waveform in HRS (blue line - sampled at $100 \mu \mathrm{s}$ ), showing multi-level fluctuations, and its value averaged over $1 \mathrm{~s}$ (red line). (c-d) Randomness distributions with (red) and without (grey) the effect of RTN considering an averaging time during readout of (c) $1 \mathrm{~s}$ and (d) $100 \mu \mathrm{s}$.

Conversely, when a short averaging time of $100 \mu$ s is selected, the average of the randomness distribution is found at about $42 \%$, limiting the reliability of the PUF circuit (Fig. 8(d)). In this framework, the compact model can be used to select the best reading scheme by trading-off the impact of RTN with time and power constraints. Still, advanced phenomena not yet included in the model such as defect volatility and anomalous RTN [46] could affect the performance of PUFs as well. These features will be included in future extensions of the model.

\section{Design of an RTN-based RNG Circuit}

Recently, the presence of RTN signals in RRAM devices has drawn attention, as their inherent randomness can be exploited as a possible source of entropy for RNG circuits [2425]. Nevertheless, a proper design of such circuits can be attained only by introducing suitable compact models, as the one proposed in this work. The circuit topology we explore (Fig. 9(a)) finds implementation in high reliability systems for the generation of truly random numbers [24]. The circuit, composed of the RRAM device in HRS and a series transistor, a buffer with a high-pass filter, and a Schmitt trigger, has been implement in Cadence Virtuoso ${ }^{\mathrm{TM}}$. We included an RRAM device initialized in HRS, with $t_{b}=1 \mathrm{~nm}$. The compact device model used here [32-33] has been extended with the proposed compact RTN model. The results of a transient simulation are shown in Fig. 9(b-c). The application of a constant voltage, $\mathrm{V}_{\mathrm{READ}}$, to the top electrode of the RRAM causes voltage RTN fluctuations to appear at the buffer input (Fig. 9(b)). The RTN pattern is transferred to the high-pass filter (to get rid of unwanted DC and low frequency components) and is fed to the Schmitt trigger. The reference voltage $V_{\text {REF }}$ regulates the amount of hysteresis that the comparator can withstand. The randomness in the RTN signal produces a random bit stream at the output of the comparator (Fig. 9(c)), achieving a noteworthy randomness value of nearly $50 \%$. The output voltage can be fed to analog-to-digital converters for further processing. The simulation of this circuit topology shows the 


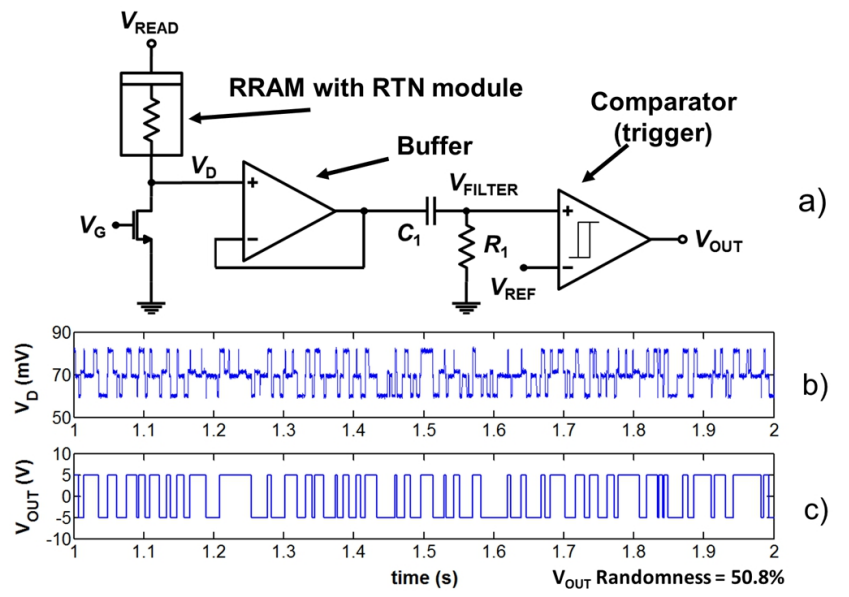

Fig. 9 - (a) Schematic of the Random Number Generator circuit based on the RTN in the RRAM device. A device in HRS with $t_{b}=1 \mathrm{~nm}$ is considered. The compact device model used here is found in [32-33] and has been extended with the proposed RTN module. (b) RTN voltage fluctuations as detected at the transistor drain terminal. (c) The random RTN pattern is reproduced at the circuit output, giving a random stream with an excellent randomness $(50.8 \%)$.

potential of the proposed model for advanced circuit design. For instance, the model can be used to estimate the best operating conditions for the RRAM device to optimize the randomness and the uniqueness of the output bit stream.

\section{CONCLUSIONS}

In this paper, we developed a physics-based compact model for RTN in RRAM, valid in both HRS and LRS, by combining refined physics-based simulations with reasonable geometrical simplifications. The model correctly considers the complex physical mechanisms at the basis of RTN in both resistive states. Its predictions are validated on a wide experimental dataset retrieved by measuring many devices in different conditions. Moreover, the model successfully captures the trends reported in the literature by other research groups. The RTN model can be seamlessly integrated into RRAM compact models. The potential of the model as a tool for advanced circuit design is clarified by showing how it can be used to $i$ ) design RTN-resilient multi-bit memory cells, ii) choose the optimal reading scheme to minimize RTN effects on RRAMbased PUF circuits, iii) design an RTN-based RNG circuit.

\section{REFERENCES}

[1] F. Crupi, R. Degraeve, A. Kerber, D. H. Kwak, and G. Groeseneken, "Correlation between Stress-Induced Leakage Current (SILC) and the $\mathrm{HfO}_{2}$ bulk trap density in a $\mathrm{SiO}_{2} / \mathrm{HfO}_{2}$ stack", Proceedings of the IEEE International Reliability Physics Symposium, pp. 181-187, 2004. doi: 10.1109/RELPHY.2004.1315321.

[2] M. Masuduzzaman, A. E. Islam, and M. A. Alam, "A multi-probe correlated bulk defect characterization scheme for ultra-thin high-K dielectric", Proceedings of the IEEE International Reliability Physics Symposium (IRPS), pp.1069-1072, 2-6 May 2010. doi: 10.1109/IRPS.2010.5488673.

[3] N. Raghavan, X. Wu, M. Bosman, and K. L. Pey, "Feasibility of SILC Recovery in Sub-10- $\AA$ EOT Advanced Metal Gate-High- $\kappa$ Stacks", IEEE Electron Device Letters, vol.34, no.8, pp.1053,1055, Aug. 2013. doi: 10.1109/LED.2013.2268246.

[4] A. Kerber, and T. Nigam, "Challenges in the characterization and modeling of BTI induced variability in metal gate / High-k CMOS technologies", Proceedings of the IEEE International Reliability Physics Symposium (IRPS), pp.2D.4.1-2D.4.6, 14-18 April 2013. doi: 10.1109/IRPS.2013.6531959.
[5] S. Mukhopadhyay, K. Joshi, V. Chaudhary, N. Goel, S. De, R. K. Pandey, K. V. R. M. Murali, S. Mahapatra, "Trap Generation in IL and HK layers during BTI / TDDB stress in scaled HKMG $\mathrm{N}$ and $\mathrm{P}$ MOSFETs", Proceedings of the IEEE International Reliability Physics Symposium (IRPS), pp.GD.3.1-GD.3.11, 1-5 June 2014. doi: 10.1109/IRPS.2014.6861146.

[6] T. Grasser, K. Rott, H. Reisinger, M. Waltl, J. Franco, and B. Kaczer, "A unified perspective of RTN and BTI", Proc. of IEEE International Reliability Physics Symposium, Waikoloa, HI, pp. 4A.5.1-4A.5.7, 2014. doi: 10.1109/IRPS.2014.6860643.

[7] J. P. Campbell, J. Qin, K.P. Cheung, L.C. Yu, J.S. Suehle, A. Oates, K. Sheng, "Random telegraph noise in highly scaled nMOSFETs", Proceedings of the IEEE International Reliability Physics Symposium (IRPS), pp.382-388, 26-30 Apr. 2009. doi: 10.1109/IRPS.2009.5173283.

[8] R. Thamankar, F.M. Puglisi, A. Ranjan, N. Raghavan, K. Shubhakar, J. Molina, L. Larcher, A. Padovani, P. Pavan, S.J. O'Shea, K.L. Pey, "Localized characterization of charge transport and random telegraph noise at the nanoscale in $\mathrm{HfO}_{2}$ films combining scanning tunneling microscopy and multi-scale simulations", (2017) Journal of Applied Physics, 122 (2), 024301. doi:10.1063/1.4991002.

[9] F. M. Puglisi, L. Larcher, P. Pavan, A. Padovani, and G. Bersuker, "Instability of HfO2 RRAM devices: Comparing RTN and cycling variability", Proceedings of the IEEE International Reliability Physics Symposium (IRPS), pp.MY.5.1-5, June 2014. doi: 10.1109/IRPS.2014.6861160.

[10] F. M. Puglisi, P. Pavan, A. Padovani, L. Larcher, and G. Bersuker, "RTS noise characterization of HfOx RRAM in high resistive state", SolidState Electronics, vol. 84, pp. 160-166, June 2013. doi: 10.1016/j.sse.2013.02.023.

[11] M. L. Fan, V. P. H. Hu, Y. N. Chen, P. Su and C. T. Chuang, "Impacts of single trap induced random telegraph noise on finfet devices and SRAM cell stability", IEEE International SOI Conference (SOI), pp. 12, Tempe, AZ, 2011. doi: 10.1109/SOI.2011.6081676.

[12] M. Si, N. J. Conrad, S. Shin, J. Gu, J. Zhang, M. A. Alam, P. D. Ye, "Low-Frequency Noise and Random Telegraph Noise on Near-Ballistic III-V MOSFETs", in IEEE Transactions on Electron Devices, vol. 62, no. 11, pp. 3508-3515, Nov. 2015. doi: 10.1109/TED.2015.2433921.

[13] L. Larcher, F. M. Puglisi, P. Pavan, A. Padovani, L. Vandelli, and G. Bersuker, "A Compact Model of Program Window in $\mathrm{HfO}_{\mathrm{x}}$ RRAM Devices for Conductive Filament Characteristics Analysis", IEEE Transactions on Electron Devices, vol.61, no.8, pp.2668-2673, Aug. 2014. doi: 10.1109/TED.2014.2329020.

[14] E. Vianello, O. Thomas, M. Harrand, S. Onkaraiah, T. Cabout, B. Traoré, T. Diokh, H. Oucheikh, L. Perniola, G. Molas, P. Blaise, J. F. Nodin, E. Jalaguier, B. De Salvo, "Back-end 3D integration of HfO2based RRAMs for low-voltage advanced IC digital design", Proc. of IEEE International Conference on IC Design \& Technology (ICICDT), pp. 235-238, Pavia, 29-31 May 2013. doi: 10.1109/ICICDT.2013.6563344.

[15] M.-C. Hsieh, Y.-C. Liao,Y.-W. Chin, C.-H. Lien, T.-S. Chang, Y.-D. Chih, S. Natarajan, M.-J. Tsai, Y.-C. King, C. J. Lin, "Ultra high density $3 \mathrm{D}$ via RRAM in pure $28 \mathrm{~nm}$ CMOS process", IEEE International Electron Devices Meeting (IEDM) Tech. Digest, pp. 10.3.1-10.3.4, 2013. doi: 10.1109/IEDM.2013.6724600.

[16] X. P. Wang, Z. Fang, X. Li, B. Chen, B. Gao, J. F. Kang, Z. X. Chen, A. Kamath, N. S. Shen, N. Singh, G. Q. Lo, and D. L. Kwong, "Highly compact $1 \mathrm{~T}-1 \mathrm{R}$ architecture (4F $\mathrm{F}^{2}$ footprint) involving fully CMOS compatible vertical GAA nano-pillar transistors and oxide-based RRAM cells exhibiting excellent NVM properties and ultra-low power operation", IEEE International Electron Devices Meeting (IEDM) Tech. Digest, pp. 20.6.1-20.6.4, San Francisco, CA, 2012. doi: 10.1109/IEDM.2012.6479082.

[17] S. Park, H. Kim, M. Choo, J. Noh, A. Sheri, S. Jung, K. Seo, J. Park, S. Kim, W. Lee, J. Shin, D. Lee, G. Choi, J. Woo, E. Cha, J. Jang, C. Park, M. Jeon, B. Lee, B. H. Lee, H. Hwang, "RRAM-based synapse for neuromorphic system with pattern recognition function", IEEE Proc. of International Electron Device Meeting, pp.10.2.1-4, 10-13 Dec. 2012. doi: 10.1109/IEDM.2012.6479016.

[18] Z. Chen, B. Gao, Z. Zhou, P. Huang, H. Li, W. Ma, D. Zhu, L. Liu, and $\mathrm{X}$. Liu, "Optimized learning scheme for grayscale image recognition in a RRAM based analog neuromorphic system", IEEE International Electron Devices Meeting (IEDM) Tech. Digest, pp. 17.7.1-17.7.4, Washington, DC, USA, 2015. doi: 10.1109/IEDM.2015.7409722. 
[19] S. Yu, B. Gao, Z. Fang, H. Yu, J. Kang, and H. S. P. Wong, "A neuromorphic visual system using RRAM synaptic devices with Sub-pJ energy and tolerance to variability: Experimental characterization and large-scale modeling", IEEE International Electron Devices Meeting (IEDM) Tech. Digest, pp. 10.4.1-10.4.4, San Francisco, CA, 2012. doi: 10.1109/IEDM.2012.6479018.

[20] I. T. Wang, Y. C. Lin, Y. F. Wang, C. W. Hsu and T. H. Hou, "3D synaptic architecture with ultralow sub-10 fJ energy per spike for neuromorphic computation", IEEE International Electron Devices Meeting (IEDM) Tech. Digest, pp. 28.5.1-28.5.4, San Francisco, CA, 2014. doi: 10.1109/IEDM.2014.7047127.

[21] A. Chen, "Utilizing the variability of resistive random access memory to implement reconfigurable physical unclonable functions", IEEE Electron Device Letters, vol.36, no.2, pp.138-140, Feb. 2015. doi: 10.1109/LED.2014.2385870.

[22] R. Liu, H. Wu, Y. Pang, H. Qian and S. Yu, "Experimental Characterization of Physical Unclonable Function Based on $1 \mathrm{~kb}$ Resistive Random Access Memory Arrays", in IEEE Electron Device Letters, vol. 36, no. 12, pp. 1380-1383, Dec. 2015. doi 10.1109/LED.2015.2496257.

[23] A. Chen, "Comprehensive assessment of RRAM-based PUF for hardware security applications", Proc. of IEEE International Electron Devices Meeting (IEDM), pp. 10.7.1-10.7.4, Washington D.C., USA, 2015. doi: 10.1109/IEDM.2015.7409672.

[24] J. Yang, J. Xu, B. Wang, X. Xue, R. Huang, Q. Zhou, J. Wu, Y. Lin, "A low cost and high reliability true random number generator based on resistive random access memory", 2015 IEEE 11th International Conference on ASIC (ASICON), Chengdu, 2015, pp. 1-4. doi 10.1109/ASICON.2015.7516996.

[25] T. Figliolia, P. Julian, G. Tognetti and A. G. Andreou, "A true Random Number Generator using RTN noise and a sigma delta converter," 2016 IEEE International Symposium on Circuits and Systems (ISCAS), Montreal, QC, 2016, pp. 17-20. doi: 10.1109/ISCAS.2016.7527159.

[26] L. S. Chang, C. Y. Huang, Y. H. Tseng, Y. C. King and C. J. Lin, "Temperature Sensing Scheme Through Random Telegraph Noise in Contact RRAM," in IEEE Electron Device Letters, vol. 34, no. 1, pp. 12-14, Jan. 2013. doi: 10.1109/LED.2012.2226137.

[27] B. Guan and J. Li, "A compact model for RRAM including random telegraph noise," 2016 IEEE International Reliability Physics Symposium (IRPS), Pasadena, CA, 2016, pp. MY-5-1-MY-5-4. doi: 10.1109/IRPS.2016.7574621

[28] S. Ambrogio, S. Balatti, A, Cubeta, A, Calderoni, N. Ramaswamy, D. Ielmini, "Statistical Fluctuations in HfOx Resistive-Switching Memory: Part II-Random Telegraph Noise“, IEEE Trans. on Electron Devices, vol. 61, n. 8, pp. 2920-2927, 2014. doi: 10.1109/TED.2014.2330202.

[29] F. M. Puglisi, P. Pavan, and L. Larcher, "Random telegraph noise in HfOx Resistive Random Access Memory: From physics to compact modeling", 2016 IEEE International Reliability Physics Symposium (IRPS), Pasadena, CA, 2016, pp. MY-8-1-MY-8-5. doi: 10.1109/IRPS.2016.7574624.

[30] F. M. Puglisi, L. Larcher, A. Padovani and P. Pavan, "A Complete Statistical Investigation of RTN in HfO2-Based RRAM in High Resistive State," in IEEE Transactions on Electron Devices, vol. 62, no. 8, pp. 2606-2613, Aug. 2015. doi: 10.1109/TED.2015.2439812.

[31] F. M. Puglisi, N. Zagni, L. Larcher and P. Pavan, "A new verilog-A compact model of random telegraph noise in oxide-based RRAM for advanced circuit design", 2017 47th European Solid-State Device Research Conference (ESSDERC), Leuven, 2017, pp. 204-207. doi: 10.1109/ESSDERC.2017.8066627.

[32] F. M. Puglisi, L. Larcher, G. Bersuker, A. Padovani, and P. Pavan, "An empirical model for RRAM resistance in low-and high-resistance states", IEEE Electron Device Letters, vol.34, no.3, p.387, Mar. 2013. doi: 10.1109/LED.2013.2238883.

[33] F. M. Puglisi, P. Pavan, A. Padovani, and L. Larcher, "A compact model of hafnium-oxide-based resistive random access memory", Proc. of IEEE International Conference on IC Design \& Technology (ICICDT), pp. 85-88, Pavia, 29-31 May 2013. doi: 10.1109/ICICDT.2013.6563309.

[34] Z. Jiang, Y. Wu, S. Yu, L. Yang, K. Song, Z. Karim, and H.-S. P. Wong, "A Compact Model for Metal-Oxide Resistive Random Access Memory With Experiment Verification," in IEEE Transactions on Electron Devices, vol. 63, no. 5, pp. 1884-1892, May 2016. doi: 10.1109/TED.2016.2545412.

[35] P. Y. Chen and S. Yu, "Compact Modeling of RRAM Devices and Its Applications in 1T1R and 1S1R Array Design," in IEEE Transactions on
Electron Devices, vol. 62, no. 12, pp. 4022-4028, Dec. 2015. doi: 10.1109/TED.2015.2492421.

[36] J. F. Kang, P. Huang, Z. Chen, Y.D. Zhao, C. Liu, R.Z. Han, L.F. Liu, X.Y. Liu, Y.Y. Wang, B. Gao, "Physical understanding and optimization of resistive switching characteristics in oxide-RRAM," 2016 46th European Solid-State Device Research Conference (ESSDERC), Lausanne, 2016, pp. 154-159. doi: 10.1109/ESSDERC.2016.7599610.

[37] F. M. Puglisi, and P. Pavan, "RTN analysis with FHMM as a tool for multi-trap characterization in HfOx RRAM", Proc. of IEEE International Conference on Electron Devices and Solid-State Circuits (EDSSC) 2013, pp. 1-2, 3-5 Jun. 2013. doi: 10.1109/EDSSC.2013.6628059.

[38] F. M. Puglisi, P. Pavan, "Factorial Hidden Markov Model analysis of Random Telegraph Noise in Resistive Random Access Memories", ECTI Transactions on Electrical Engineering, Electronics, and Communications, vol. 12, no.1, pp. 24-29, 2014.

[39] MDLsoft Inc. - 02/17/2018 - http://www.mdlsoft.com

[40] L. Vandelli, A. Padovani, L. Larcher, R. G. Southwick III, W. B. Knowlton, and G. Bersuker, "A Physical Model of the Temperature Dependence of the Current Through $\mathrm{SiO}_{2} / \mathrm{HfO}_{2}$ Stacks", IEEE Transactions on Electron Devices, vol.58, no.9, pp.2878-2887, Sept. 2011. doi: 10.1109/TED.2011.2158825.

[41] D. Veksler, G. Bersuker, L. Vandelli, A. Padovani, L. Larcher, A. Muraviev, B. Chakrabarti, E. Vogel, D. C. Gilmer, P. D. Kirsch, "Random telegraph noise (RTN) in scaled RRAM devices", Proceedings of the IEEE International Reliability Physics Symposium (IRPS), MY.10.1-4, Apr. 2013. doi: 10.1109/IRPS.2013.6532101.

[42] G. Bersuker, D. C. Gilmer, D. Veksler, P. D. Kirsch, L. Vandelli, A. Padovani, L. Larcher, K. McKenna, A. Shluger, V. Iglesias, M. Porti, M. Nafria, "Metal oxide resistive memory switching mechanism based on conductive filament microscopic properties", Journal of Applied Physics, vol. 110, p. 124518, 2011. doi: 10.1063/1.3671565.

[43] F. M. Puglisi, P. Pavan, L. Vandelli, A. Padovani, M. Bertocchi, L. Larcher, "A microscopic physical description of RTN current fluctuations in HfOx RRAM", Proc. of IEEE International Reliability Physics Symposium (IRPS), pp. 5B.5.1-5B.5.6, Monterey, CA, 19-23 April 2015. doi: 10.1109/IRPS.2015.7112746.

[44] S. Ambrogio, S. Balatti, A. Cubeta, A. Calderoni, N. Ramaswamy, D. Ielmini, "Understanding switching variability and random telegraph noise in resistive RAM", IEEE Proc. of IEEE Proc. of International Electron Device Meeting, pp. 31.5.1-4, 9-11 Dec. 2013. doi: 10.1109/IEDM.2013.6724732.

[45] N. Raghavan, R. Degraeve, A. Fantini, L. Goux, S. Strangio, B. Govoreanu, D.J. Wouters, G. Groeseneken, M. Jurczak, "Microscopic origin of random telegraph noise fluctuations in aggressively scaled RRAM and its impact on read disturb variability", Proc. of IEEE International Reliability Physics Symposium (IRPS), pp. 5E.3.1-5E.3.7, Anaheim, CA, April 2013. doi: 10.1109/IRPS.2013.6532042.

[46] T. Grasser, "Stochastic charge trapping in oxides: from random telegraph noise to bias temperature instabilities", Microelectronics Reliability, vol. 52 p. 39-70, 2009. doi: 10.1016/j.microrel.2011.09.002.

[47] J. Robertson, R. Gillen, "Defect densities inside the conductive filament of RRAMs", Microelectronic Engineering, vol. 109, pp. 208-210, Sept. 2013, ISSN 0167-9317. doi: 10.1016/j.mee.2013.03.010.

[48] S. Balatti, S. Ambrogio, A. Cubeta, A. Calderoni, N. Ramaswamy and D. Ielmini, "Voltage-dependent random telegraph noise (RTN) in HfOx resistive RAM," 2014 IEEE International Reliability Physics Symposium, Waikoloa, HI, 2014, pp. MY.4.1-MY.4.6. doi: 10.1109/IRPS.2014.6861159.

[49] F. M. Puglisi, P. Pavan, L. Larcher and A. Padovani, "Analysis of RTN and cycling variability in HfO2 RRAM devices in LRS", 2014 44th European Solid State Device Research Conference (ESSDERC), Venice, 2014, pp. 246-249. doi: 10.1109/ESSDERC.2014.6948806.

[50] F. M. Puglisi, P. Pavan, L. Larcher, and A. Padovani, "Statistical analysis of random telegraph noise in HfO2-based RRAM devices in LRS", Solid-State Electronics, Volume 113, 2015, Pages 132-137, ISSN 0038-1101. doi: 10.1016/j.sse.2015.05.027.

[51] F. Lamarche, and C. Leroy, "Evaluation of the volume of intersection of a sphere with a cylinder by elliptic integrals", Computer Physics Communications, Volume 59, Issue 2, 1990, Pages 359-369, ISSN 0010-4655. doi: 10.1016/0010-4655(90)90184-3.

[52] A. Padovani, L. Larcher, G. Bersuker and P. Pavan, "Charge Transport and Degradation in $\mathrm{HfO}_{2}$ and $\mathrm{HfO}_{x}$ Dielectrics", in IEEE Electron Device Letters, vol. 34, no. 5, pp. 680-682, May 2013. doi: 10.1109/LED.2013.2251602. 